\title{
DRIMPAC-Unified Demand Response Interoperability Framework Enabling Market Participation of Active Energy Consumers ${ }^{+}$
}

\author{
Dimitrios Tzovaras ${ }^{1}$, Ioannis Damousis ${ }^{1}$, Antonis Papanikolaou ${ }^{2}$, Giorgos Pitsiladis ${ }^{2}$ and \\ Giulia Barbagelata ${ }^{3, *}$ \\ 1 Center for Research and Technology Hellas, Information Technologies Institute, 57001 Thessaloniki, \\ Greece; dimitrios.tzovaras@iti.gr (D.T.); damousis@iti.gr (I.D.) \\ 2 Hypertech SA, 32152 Athens, Greece; a.papanikolaou@hypertech.gr (A.P.); g.pitsiladis@hypertech.gr (G.P.) \\ 3 Stam S.r.l., 16121 Genova, Italy \\ * Correspondence: g.barbagelata@stamtech.com \\ † Presented at the Sustainable Places 2019 (SP 2019), Cagliari, Italy, 5-7 June 2019. \\ Published: 24 July 2019
}

\begin{abstract}
Residential and tertiary buildings are responsible for $44 \%$ of final energy consumption in Europe. However, they are currently not engaged in Demand Response (DR) activities due to technology-related and consumer-related roadblocks. One of the main technological roadblocks is the extreme fragmentation of protocols, data models and standards for Building Energy Management (BEM) systems and Building to Grid (B2G) communications. DRIMPAC is an EUfunded Innovation Action that aims to address the interoperability gaps and standards fragmentation in the residential and commercial buildings Demand Response domain aiming to reduce costs for all involved actors and make DR more attractive for the end prosumer.
\end{abstract}

Keywords: demand response; standards; OpenADR; IEC61850; residential; tertiary buildings

\section{Introduction}

Extreme fragmentation of protocols, data models and standards for Building Energy Management (BEM) systems and Building to Grid (B2G) communications leads to very high cost for the energy retailer or aggregator for customer acquisition and interfacing with utilities for small DR customers. Consumers are also reluctant to participate because current implicit DR programs are often obtrusive and carry the risk of increased bills or inconvenience while consumers are also riskaverse [1] due to the criticality of the commodity (electricity), unless there are clear benefits for them, the reassurance of reliability and the sense of system ownership. If these roadblocks are overcome, massive demand flexibility potential from distributed buildings can potentially be unleashed, leading to higher RES penetration in the energy mix and deferring costly grid infrastructure investments. To achieve this transformation and materialize the benefits of demand response for the entire energy system, retailers will require three main elements:

- Technological foundation: the IT infrastructure for end-to-end interoperability among the systems of all involved actors, the BEMS \& automation components.

- Market push: innovative and value-adding propositions for the customers that build upon digital, smart services and devices to deliver truly engaging and rewarding experiences for consumers.

- Energy consumer pull: human-centric technologies, prioritizing personal comfort and lifestyle preferences along with energy costs and energy system reliability in order to 
maintain the consumer engagement and prolonged participation in DR programs and convincing demonstration of these innovative propositions for creating an initial critical mass of early adopters, opinion leaders and credible success stories.

DRIMPAC [2] aims to enable small prosumers (building level) to participate in implicit and explicit demand response programs (see Figure 1) by: addressing the interoperability gaps and standards fragmentation by developing an end-to-end solution bridging major standards (OpenADR, OneM2M, IEC 61850...), while striking the right balance between comfort preservation and energy conservation via environmental and preferences monitoring and intelligent algorithms; Supporting multiple building topologies covering over $90 \%$ of building stock and $40 \%$ of final energy use in Europe and various energy mixes (electricity, gas, district heating) for a holistic optimization framework; Leveraging local generation and storage infrastructure (self-consumption, load shifting, thermal inertia) taking into account the profiles of the end-users and their (lack of) technical expertise.

The expected benefits include potentially lower energy usage and costs for the occupants, possibly making a profit in the long-run, increased self-consumption, peak shaving and shifting benefits for the DSO, potential auxiliary services benefits for the DSO leading to new business opportunities for aggregators, building managers, retailers.

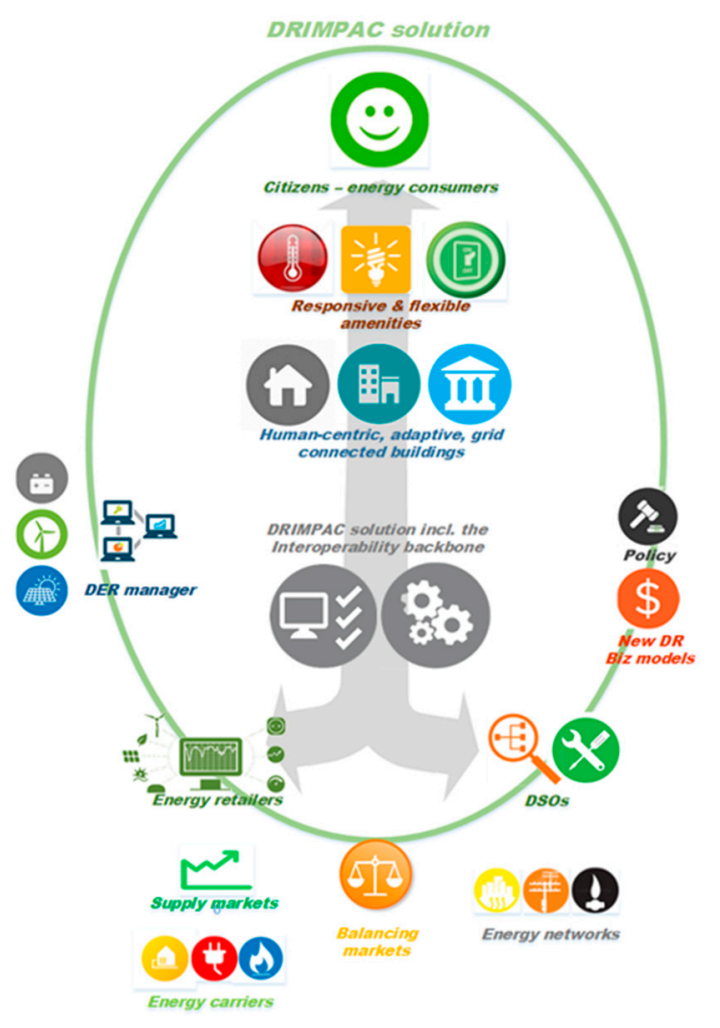

Figure 1. The DRIMPAC solution.

The DRIMPAC solution will be tested in four pilot sites in Spain, Cyprus, Germany and France, covering a representative range of climate and building types (campus, commercial, offices, residencies) in the EU, involving more than 1000 end-users. In this paper the project concept is presented, as well as the planned pilot deployment activities.

\section{DRIMPAC Concept}

The DRIMPAC solution is an ICT framework that will facilitate the open, standards-based, efficient and cost-effective massive roll-out of demand response programs supporting both price- and incentive-based DR schemes. It comprises two main service layers: The interoperability layer and the automation layer. 


\subsection{The Interoperability Layer}

The interoperability layer or backbone will establish the required end-to-end, bi-directional, multi-purpose communication channels between the energy retailer or aggregator and the building infrastructure based on open standards to enable interoperability, vendor lock-in avoidance and easy switching between suppliers. These channels and the associated data models and protocols are critical for the mass scale deployment of implicit and explicit demand response programs for multiple energy carriers by enabling information exchange about energy prices, grid requests for demand flexibility as well as supporting information like demand flexibility potential and measurements for optimal demand profile management.

Two main gaps need to be bridged for end-to-end interoperability. The first gap and main challenge lies on the extreme fragmentation of the supervisory control and automation solutions available within buildings and the diversity of standards they are based on, or lack thereof. These solutions are broadly divided into the rather mature Building Management Systems (BMS) for the tertiary building market and Smart Home solutions/ products for residential buildings. BMSs are more established and supported by a handful of standards - e.g., BAC, KNX, Lon - for which intrabuilding interoperability solutions already exist. The Smart Home situation is very different. The market has not reached maturity and the available solutions rely on either closed standards-e.g., ZWave, ZigBee profiles - or more popularly on proprietary interfaces-e.g., IP-based proprietary web service APIs. Given the market immaturity, it is very risky to anticipate which standard/protocol will dominate, if any. Standardization bodies are going in another direction and establishing top-down standards to facilitate Smart Home communications (oneM2M is the prime example developed by ETSI/CEN/CENELEC and actively supported by the EC (Figure 2)). Unfortunately this standard is not supported by any of the existing products and it will take years before it penetrates the market.

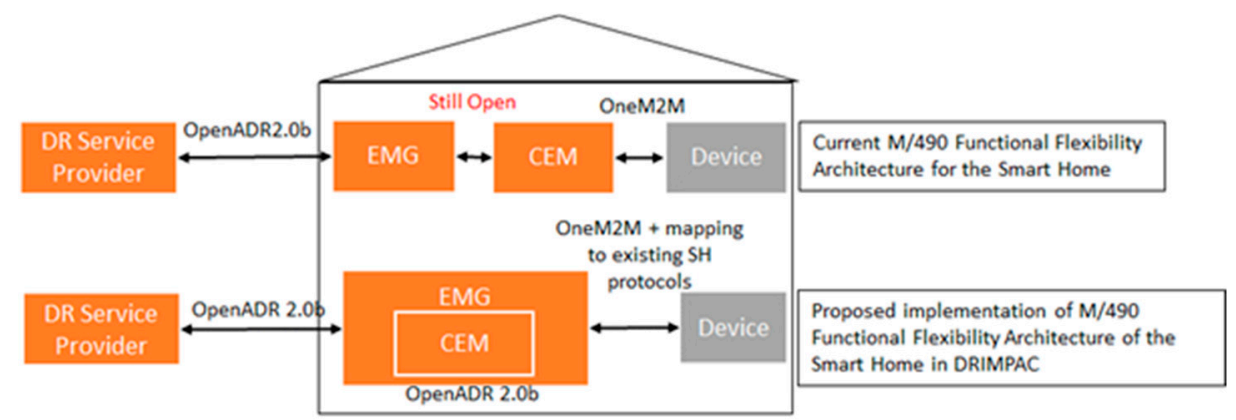

Figure 2. $M / 490$ Functional Flexiblity Architecture and the DRIMPAC proposal to enable interoperability with existing Smart Home products.

The second gap for end-to-end interoperability involves the communications between the building management system and the market actor. The main prevailing standard for demand response is OpenADR [3]. It is an open, validated U.S. standard which is currently under standardization by European and worldwide standardization bodies, e.g., ETSI, IEC, in order to become the de facto standard for DR communications in the electricity grid. The DRIMPAC solution will leverage these upcoming standards to propose a unified, future-proof interoperability framework together with the necessary components to interface with devices already in the field. Furthermore, this framework will be extended to support DR communications to district-level Distributed Energy Resources - e.g., generators, storage - in order to provide a unified interface that gives market actors access to a huge portfolio of distributed energy demand sources.

\subsection{The Automation Layer}

The automation layer aims to optimize building operations with the primary objective to reduce energy costs while maintaining comfortable and healthy living/working indoor conditions for the occupants. It will maximise savings by facilitating human-centric, automated building demand adaptation without relying on consumers to manually manage loads, albeit they will always maintain 
opt-out options in order to preserve the final say in the building operations. This layer will host the necessary capabilities to infer user comfort profiles as well as automate the definition and application of human-centric optimal load control strategies that minimise costs and/or energy savings while preserving the comfort and air quality aspects of the indoor environment. Taking advantage of dynamic energy prices and explicit DR requests, the automation layer will modify the building energy demand profile of the appropriate energy carrier accordingly. The availability of local generation for self-consumption or energy storage that can increase demand flexibility will be factored in the optimization to maximize consumer benefits.

\section{DRIMPAC Pilot Sites}

The main objectives of the pilot activities will be to:

- Demonstrate the seamless integration and interoperability of various building typologies and underlying assets into the DRIMPAC Energy Demand Management Framework which aims to leverage flexible electricity demand to increase the impact and value of DR programs;

- evaluate the performance of the Building monitoring and Automation Layer to discover and automatically exploit demand flexibility without degrading the occupants' comfort; and

- validate the associated service offering and business models, analyse consumer acceptance in real-life conditions and assess their commercial viability.

The pilot validation activities will be hosted by two small and two medium-sized energy retailers in four countries-involving various energy use cultures, market and climatic conditions. Each pilot site will comprise buildings of both residential and tertiary use that are direct customers of the retailers that participate in the consortium ensuring direct access to the building owners/managers/dwellers

\subsection{French (Saint-Julien Montdenis) Demonstration Site Description}

\subsubsection{Description of Facilities}

The French pilot site will comprise both tertiary (offices) and residential buildings. The office building was constructed in 2010 using low-energy consumption principles and techniques and is powered by electricity only. A series of sensors (e.g., light, temperature, presence) are already installed in the building from previous research activities. The total annual energy consumption of the building is approximately $80 \mathrm{MWh}$. In addition, two multi-apartment residential buildings will take part in the pilot. The first is a purely residential, three story building with 45 apartments. The building was renovated in 2014 and is purely electricity powered, including space and water heating. The total annual electricity consumption is $270 \mathrm{MWh}$. The second has three floors housing 45 apartments and on its ground level it also hosts 11 shops. The building consumes about $340 \mathrm{MWh}$ annually. During pilot demonstration activities, the lower residential floor of both buildings will be used as the control group, while project interventions will take place on the top two floors. The office building is equipped with $75 \mathrm{~kW}$ rooftop solar panels. The building is also equipped with charging stations for two Electric Vehicles. Furthermore, a monitoring and supervisory control system based on commercial energy management system solution is used to monitor ambient conditions and user preferences. The residential buildings will be equipped with smart meters before the project interventions start.

\subsubsection{Use Cases to Be Tested}

Both price-based (implicit) and incentive-based (explicit) DR programs will be tested in the buildings. Furthermore, the availability of local intermittent generation and electricity storage facilities will enable a deep cost optimization using self-consumption principles. Furthermore, 
various innovative dynamic electricity tariffs will be tested to evaluate their impact of consumer behaviour and achievable cost/comfort benefits.

\subsection{Cypriot (Nicosia) Demonstration Site Description}

\subsubsection{Description of Facilities}

The University of Cyprus (UCY) campus comprises 17 tertiary buildings that span a broad variety of typologies and uses. They range from educational facilities (classrooms, amphitheaters), office building, restaurants, sports and health centres, etc. They are all new constructions, from 2008 onwards, and are energy efficient buildings. These buildings are used by more than 1500 personnel and many hundreds of students on a daily basis. UCY operates a district heating grid to serve the building heating/cooling needs. All buildings combined annually consume about $7800 \mathrm{MWh}$ of electricity as an end-use and 15,700 MWh of heat/cold. All buildings are equipped with building management systems from various vendors. The campus possesses significant generation capacity. Several rooftop PV installations are already operational in campus buildings and a solar park of 10 MWp is under commissioning within the campus. UCY operates the campus (electricity and district heating/cooling) network as a micro-grid and has installed a central energy management system overseeing buildings operations including with supervisory control capabilities.

\subsubsection{Use Cases to Be Tested}

Versatile DR use cases will be tested in UCY: a) the achievable benefits from deploying dynamic electricity tariffs and explicit DR schemes will be validated on buildings with various purposes, occupancy patterns and comfort preferences; $b$ ) the DRIMPAC part dedicated to managing districtlevel DER systems will be extensively tested in this pilot; c) self-consumption at the campus level and its cost optimization impact will be evaluated; and d) the remote management of building loads by energy managers deployed centrally can be tested.

\subsection{German (Trier) Demonstration Site Description}

\subsubsection{Description of Facilities}

SWT is a German municipal supplier of electricity, gas, district heating and water, responsible for a region of 500,000 inhabitants. SWT contributes residential and office buildings for the pilot validation activities, as well as a couple of infrastructures that will be used as a district-level DER system offering short-time storage and generation capabilities to the grid. The office building was built in 1959 and a deep renovation in planned in the short term future. The building is powered by both electricity and natural gas. The SWT office building annually consumes about $1.1 \mathrm{GWh}$ of electricity and about $1.65 \mathrm{GWh}$ of natural gas. The residential building is a four-story multifamily block with 8 apartments. The block was built in 1963 and will be renovated in 2017. Each of the housing units of the residential building have an average annual heat consumption of 5000-7390 kWh from district heating and an annual average electricity consumption of 1500-3000 kWh.

In addition to these buildings, SWT will also provide two infrastructures that can be used as district level DER systems to validate the integration of alternative DERs-beyond buildings -in the DRIMPAC solution, namely the district heating plant and a public swimming pool. The district heating plant was built in 1963, renovated and modernized in 2001 and extended in 2005 with an additional combined heat and power unit (CHP). Its new CHP unit has a total electrical capacity of 2 MW and an annual production of $9 \mathrm{GWh}$. It also has a thermal capacity of $2.4 \mathrm{MW}$ and an annual production of approx. $10.8 \mathrm{GWh}$. Further heating is supplied by two boilers with a thermal capacity of 5.25 MW and 4.25 MW and together an annual production of $7 \mathrm{GWh}$. The indoor swimming pool was renovated in 2009 with energetic measures and its saunas were constructed. The swimming pool has a usable annual heat consumption of $3.00 \mathrm{GWh}$ and an annual electricity consumption of approx. 1.5 GWh. A CHP with an electrical output of $357 \mathrm{~kW}$ and a thermal output of $529 \mathrm{~kW}$ as well as three gas boilers with a capacity of $690 \mathrm{~kW}$ are installed in the building for heat and power supply. 


\subsubsection{Use Cases to Be Tested}

The main differentiator of the German pilot site compared to the others is the involvement of multiple energy carriers in the buildings under test. The DRIMPAC system will be deployed with the aim to optimize cost across all available carriers-electricity, gas and district heating -in a humancentric manner. Dynamic energy tariffs for multiple carriers will be tested to evaluate the impact on energy consumption and ability of consumers - and automatic tools - to respond efficiently to such a scenario. In addition to this, the swimming pool building and its electricity generation facilities will be used for the validation of self-consumption schemes, by virtually combining them with the electricity loads of the buildings.

\subsection{Spanish (Murcia) Demonstration Site Description}

\subsubsection{Description of Facilities}

The Joven Futura residential neighbourhood comprises of 4 independent buildings (2006). Each building is 8 stories high and hosts 30 flats, a reception and an underground parking lot with storage space. The buildings are managed by an external facility manager and are powered by electricity with a total annual consumption of $100 \mathrm{MWh}$. The flats are all fitted with individual smart electricity meters and some properties have already installed smart thermostats. Only water heating is powered by natural gas. The Parque Científico de Murcia is an office building (2007) hosting approx. The building includes an auditorium, a restaurant, a kitchen, a coffee corner, meeting rooms, the offices and different bathrooms in each floor. The building is powered with electricity only (total annual consumption of approx. $120 \mathrm{MWh}$ ). Smart metering is applied at the building level, while several smart devices (thermostats and temperature/ luminance sensors) have been deployed. The MAGALIA Business Center is an office building (2004) consisting of a reception, one auditorium, underground parking, a restaurant, a kitchen, coffee corner, classrooms, meeting rooms, and several offices. The building is managed by an in-house facility manager and is powered by electricity only with a total annual consumption of approx. $260 \mathrm{MWh}$. Smart metering is applied at the building level, while several smart devices (thermostats and temperature/ light sensors) have already been deployed in the various building spaces.

\subsubsection{Use Cases to Be Tested}

MiWEnergia already offers dynamic electricity tariff schemes to its customers. This pilot site will spearhead the detailed investigation of how consumers respond to the various tariff schemes for electricity. Several tariff types will be tested and demonstrated, e.g., ToU, CPP, RTP, Peak Time Rebates, with varying price ratios/volatilities and number/frequency of price spikes in order to test the performance, persistence and potential fatigue of consumers with and without automation. The close relationship of MiWEnergia with its customers, compared to the other retailers of the consortium, makes them more easily accessible and open to provide more information to the consortium.

\section{Conclusions}

In this paper the high-level concept of DRIMPAC has been presented along with the pilot sites that will be used for the validation of DRIMPAC results. DRIMPAC is a three-year Innovation Action, funded by the EU, aiming to create an interoperability framework that bridges several standards and communication protocols between the DSO and the end-prosumer, in order to enable seamless Demand Response services in the residential and tertiary building domains. The flexibility potential of these buildings, in addition to district level DERs is huge and technological means such as DRIMPAC that facilitate the large scale deployment of DR are expected to have a positive impact on the stabilization of the future grids towards the maximum utilization of distributed renewable resources. The project will be tested in four sites aiming to involve more than 1000 end-users. 
Funding: This project has received funding from the European Union's Horizon 2020 Research and Innovation programme under Grant Agreement No. 768559.

Conflicts of Interest: The authors declare no conflict of interest.

\section{References}

1. Gülen, G.; Foss, M.M. Real Time Pricing in Electricity Markets. The Institute for Energy, Law \& Enterprise, University of Houston. Available online: https://pdfs.semanticscholar.org/1260/d62db313e74abc8e6c1ee8890c82bd472255.pdf?_ga=2.167676027.4320 25614.1563855813-1118834543.1544147605 (accessed on 1 June 2019).

2. DRIMPAC project website. Available online: www.drimpac-h2020.eu (accessed on 1 June 2019).

3. OpenADR 2.0 Profile Specification B Profile; Herberg, U., Zuber, J., Eds.; OpenADR Alliance: Morgan Hill, CA, USA, 2013.

(C) 2019 by the authors. Licensee MDPI, Basel, Switzerland. This article is an open access article distributed under the terms and conditions of the Creative Commons Attribution (CC BY) license (http://creativecommons.org/licenses/by/4.0/). 